\title{
Synergistic and antagonistic co-antioxidant effects of flavonoids with trolox or ascorbic acid in a binary mixture
}

\author{
LEVON A TAVADYAN*(D and SEYRAN H MINASYAN \\ Institute of Chemical Physics National Academy of Science, Republic of Armenia, 5/2, Sevak Street, \\ 0014 Yerevan, Armenia \\ E-mail: tavadyan@ichph.sci.am
}

MS received 10 December 2018; revised 6 March 2019; accepted 9 March 2019; published online 9 May 2019

\begin{abstract}
By using the ORAC method, the values of the antiperoxyradical capacity of binary mixtures of bioflavonoids (quercetin, rutin, morin and naringin) with trolox or ascorbic acid, and individual antioxidants in the aqueous medium were determined. Through these values and the data of square wave voltammetry (SWV) method, the non-additive effects in the binary mixtures were revealed. Synergy in the binary mixtures was observed in the presence of flavonoids (rutin and naringin) having O-glucosyl group in the molecular structure. Flavonoids without a glucosyl group in the molecular structure (quercetin and morin), having relatively small values of the oxidation potential and low bond dissociation enthalpy (BDE) of the most reactive $\mathrm{OH}$ group in the mixtures with trolox or ascorbic acid, have shown antagonistic effects. In this work, 'skeletal' kinetic models are considered, explaining the non-additive effect of co-antioxidants under study, conditioned by the possibility of antioxidant regeneration with high (synergy) or low (antagonism) antiradical capacity.
\end{abstract}

Keywords. Bioflavonoids; ORAC; antioxidants; antiperoxyradical capacity; synergistic effect; antagonistic effect.

\section{Introduction}

Flavonoids are widely distributed in plants, mainly in fruits, vegetables, tea, etc. ${ }^{1-4}$ They constitute a large class of compounds containing several phenolic hydroxyl groups attached to ring structures, conferring antioxidant properties. ${ }^{5-9}$ It has been established that these compounds can scavenge free radicals and other oxygen species in the body. ${ }^{3,10,11}$ Therein lie their useful properties in preventing pathologies of the oxidative stress. Flavonoids in foodstuff and biosystems act as antioxidants not separately, but in a mixture. Simultaneously, understanding chemical mechanisms of cooperative effect of antioxidants will allow one to conduct more purposeful selection of antioxidant systems for food and medicinal agents. In such cases, their non-additive cooperative effect, synergy or antagonism, may be manifested. A mixture of antioxidants exhibits high (synergistic) or low (antagonistic) effect than the sum of antioxidant effects of individual compounds taken at the same concentrations. ${ }^{12-22}$ Flavonoids are

\footnotetext{
*For correspondence
}

water-soluble and studying their antioxidant properties when mixed with similar antioxidants, trolox or ascorbic acid, is a problem of scientific and practical interest. Trolox simulates the possible synergistic antioxidant effect of a liposoluble antioxidant $\alpha$-tocopherol that acts in combination with water-soluble antioxidants most probably in the polar part of cell membranes. ${ }^{19}$

Many researches are focused on investigating the phenomenon of synergy in the mixtures of bioantioxidants including flavonoids. ${ }^{13,17-21,23-26}$ This phenomenon is essentially explained by the regeneration of the active antioxidant via aryloxyl radical of the more passive co-antioxidant, ${ }^{12,14,16,20,21}$ similar to the well-known synergy between $\alpha$-tocopherol or ascorbic acid. ${ }^{14,17,22}$ Such explanation seems to relate to the antioxidant activity of compounds, characterizing the level of their inhibiting influence on the oxidation rate of substrates. Antiradical capacity frequently reflects an antioxidant capability. Antiperoxyradical capacity is defined as the number of peroxyl radicals trapped by the molecule of an antioxidant. In such cases, to describe the nonadditive effect of co-antioxidant, a detailed account must be taken into account involving pro-oxidant reactions with participation of antioxidants and fitting intermediates as well as their kinetic behavior during induction 
period of lipids oxidation. In the present research, antiperoxyradical capacity $(f)$ is considered as the main characteristic of the antioxidant ability of the compounds under study and their binary systems without oxidizing substrates.

Peroxyl radicals are basic chain carriers at chain peroxidation of cell membrane lipids. It is common knowledge that uncontrollable intensification of the peroxidation processes of cell membrane lipids results in oxidative diseases. The compounds under consideration are regulators of lipid peroxidation, acting as chainbreaking antioxidants that trap kinetic chain carriers, the peroxyl radicals. ${ }^{4,12,14,16}$

Kinetic method of the determination of absorption capacity in relation to oxygen-centred radicals ('Oxygen Radical Absorption Capacity'- ORAC) ${ }^{26-28}$ is selected as the basis to determine the antiperoxyradical capacity. This method was selected because it takes into account the following circumstances. Integrally, the non-additive effect of a mixture of antioxidants is conditioned by their combined systemic behavior in the reaction system. The non-additive effect (synergy, antagonism) of antioxidants in many respects depends on the nature of the reaction system itself. We suggest that two types of non-additive effects of a mixture of antioxidants should be differentiated, which become apparent in i) the presence of pro-oxidant reactions that assist oxidation with the participation of antioxidants and their intermediates, and ii) the absence of prooxidant reactions with participation of antioxidants and their intermediates. This enables a step-by-step understanding of the complex mechanism of non-additive effects for the mixture of antioxidants.

In this work, along with the ORAC method, the square wave voltammetry (SWV), the electroanalytical method, was used retracing the consumption kinetics at least for one species of the mixture of antioxidants. This method was applied to facilitate the interpretation of data obtained by the ORAC method.

The work is aimed at studying the combined antioxidant effect of four structurally related flavonoids: rutin, quercetin, morin and naringin paired with ascorbic acid or trolox in the aqueous medium. Simultaneously, we studied the chemical mechanism of non-additive effects in the mixture of antioxidants under consideration. The general structure of flavonoids containing three rings, denoted by $\mathrm{A}, \mathrm{B}$ and $\mathrm{C}$ is depicted in Figure 1 . In the studied flavonoids, a pyranyl ring $\mathrm{C}$ containing a double bond, a carbonyl group and a hydroxyl or sugar groups are present. Substitution of hydroxyl groups in the A and B rings by other groups (for example, sugar group for naringin) also distinguishes individual compounds. ${ }^{7-9}$
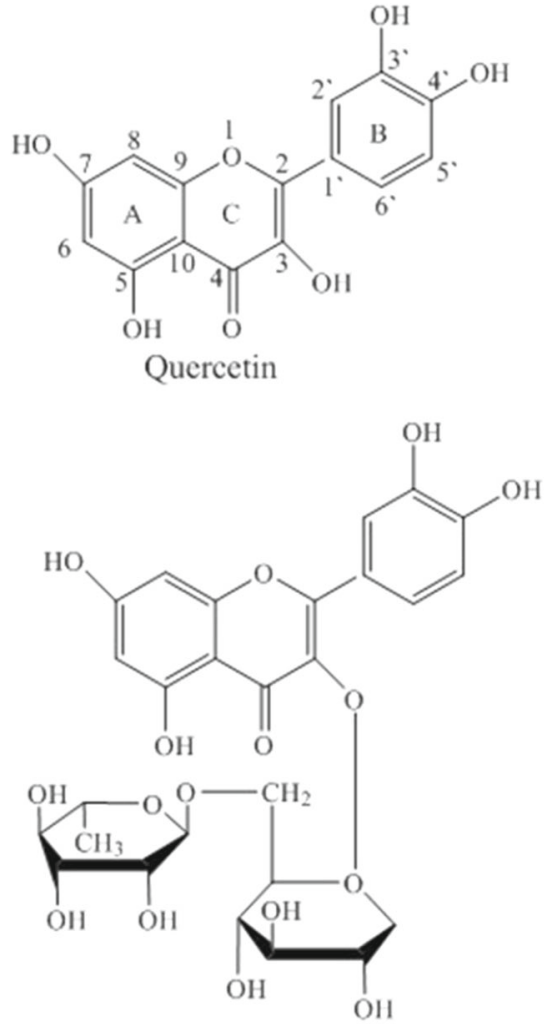

Rutin<smiles>COc1cc(O)cc2oc(-c3ccc(O)cc3O)c(O)c(=O)c12</smiles><smiles>O=c1cc(-c2ccc(O)cc2)oc2cc(OC3O[C@@H]4[C@H]5O[C@H](O[C@H](O)[C@H]5O)O[C@@H]4[C@H](O)[C@@H](CO)O3)cc(O)c12</smiles>

Naringin

Figure 1. Molecular structures of the investigated flavonoids. 


\section{Experimental}

\subsection{Materials}

2,2'-Azobis(2-amidinopropane) dihydrochloride (AAPH), 2(3, 4-dihydroxyphenyl)-5, 7-dihydroxy-3-[(2S, 3R, 4S, 5S, 6R)-3，4，5-trihydroxy-6-(2R，3R，4R，5R，6S)-3，4，5trihydroxy-6-methyloxan-2-yl]oxymethyl]oxan-2-yl]oxychr omen-4-one (Rutin), 2-(2, 4-dihydroxyphenyl)-3, 5, 7-trihydr oxychromen-4-one-2-(2, 4-di-hydroxyphenyl)-3, 5, 7-trihydr oxychromen-4-one (Morin), 2-(3, 4-di-hydroxyphenyl)-3, 5, 7-trihydroxy-4H-chromen-4-one (Quercetin), 7-[[2- $O$-(6Deoxy- $\alpha$-L-mannopyranosyl)- $\beta$-D-glucopyranosyl]oxy]-2, 3-dihydro-5-hydroxy-2-(4-hydroxyphenyl)-4H-1-benzopyr an-4-one (Naringin), 6-hydroxy-2, 5, 7, 8-tetra-methylchro man-2-carboxylic acid (trolox), ascorbic acid (AA), disodium 2.2b ORAC method: Determination of absorption capacity in relation to oxygen-centred radicals: The method is based on decrease in fluorescence intensity of fluorescein (FL) with time, conditioned by consumption of FL in the reaction with oxygen-centred radicals. ${ }^{26-28}$ Antioxidants introduced in the reaction medium interact with the radicals generated by thermal decomposition of the azo initiator, thus suppressing free radical consumption by FL. Antiradical capacity of antioxidants is determined merely on the basis of this effect.

In the ORAC method, fluorescence spectrometer PerkinElmer MPF-44B (USA) was used with computer registration of fluorescence intensity change of FL as a result of reaction with ROO peroxyl radicals. The latter were generated by thermal decomposition of a water-soluble AAPH azocompound in the presence of dioxygen, Scheme (1):

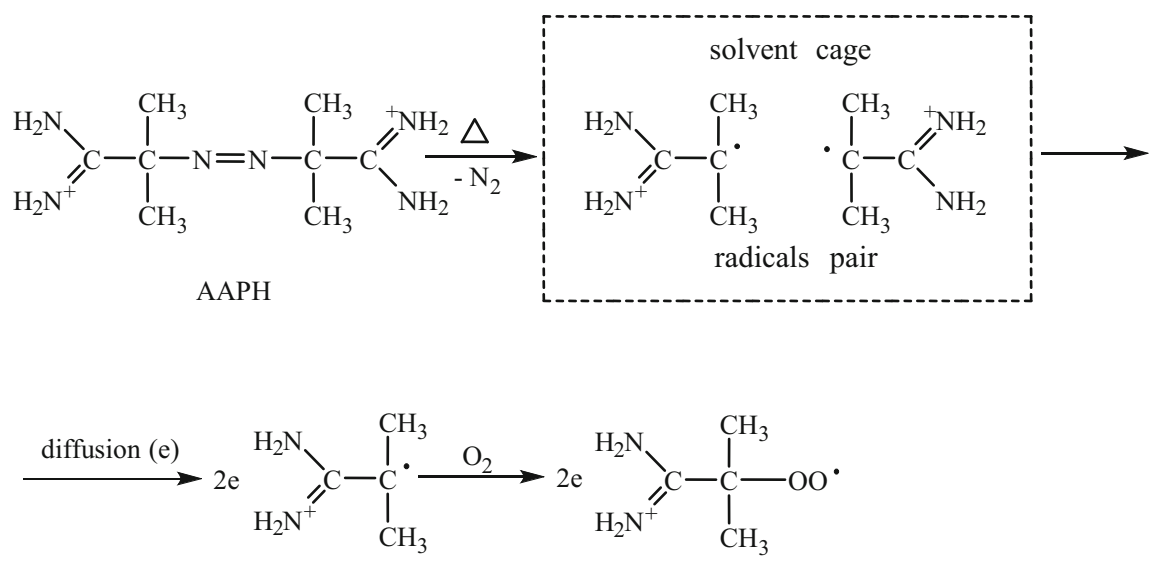

Scheme 1. Peroxyl radical generation by thermal decomposition of azocompound AAPH in the presence of dioxygen.

3-oxospiro[2-benzofuran-1, 9'-xanthene]-3', 6'-diolate (disodium salt of fluorescein (FL)), solvents (ethanol, phosphate buffer) and $\mathrm{K}_{3}\left[\mathrm{Fe}(\mathrm{CN})_{6}\right]$ were purchased from company Sigma-Aldrich (USA). Ethanol was additionally purified according to the method described in Ref. ${ }^{29}$ Deionized water with the electrical resistance of $18.2 \mathrm{MOhm} \cdot \mathrm{cm}$ at $25^{\circ} \mathrm{C}$ (purified by EASY pure II equipment, USA) was used.

\subsection{Methods}

2.2a Sample preparation: Antioxidant samples were prepared in the purified ethanol. The concentration of antioxidants in the stock solution was $4 \times 10^{-4} \mathrm{M}$. Following amounts of antioxidants were added to the reaction mixture $(3 \mathrm{~mL})$ : rutin $38 \mu \mathrm{L}\left(5 \times 10^{-6} \mathrm{M}\right)$, quercetin $38 \mu \mathrm{L}$ $\left(5 \times 10^{-6} \mathrm{M}\right)$, morin $38 \mu \mathrm{L}\left(5 \times 10^{-6} \mathrm{M}\right)$, naringin $38 \mu \mathrm{L}$ $\left(5 \times 10^{-6} \mathrm{M}\right)$, ascorbic acid $38 \mu \mathrm{L}\left(5 \times 10^{-6} \mathrm{M}\right)$ and trolox $38 \mu \mathrm{L}\left(5 \times 10^{-6} \mathrm{M}\right)$. The mixtures of antioxidants were prepared by mixing individual solutions of two antioxidants taken in volumes of $38 \mu \mathrm{L}\left(5 \times 10^{-6} \mathrm{M}\right)$. The concentrations of the initiator of peroxyl radicals, AAPH, and fluorescein in the reaction mixture were $1.73 \times 10^{-3} \mathrm{M}$ and $5 \times 10^{-7} \mathrm{M}$, respectively. Studies were carried out in aqueous solution (phosphate buffer 0.1 M, pH = 7.4). where $e$ is the yield of the formed radicals from the solvent cage according to the reaction. The excitation and emission wavelengths were 450 and $515 \mathrm{~nm}$, respectively. The antiperoxyradical capacity of the compounds under study was quantitatively determined from the area adjacent between two kinetic curves on the fluorescence intensity decrease of FL with and without antioxidant addition (under conditions of FL full consumption). Trolox was used as a standard antioxidant. The value of antiperoxyradical capacity $f_{\mathrm{AO}}$, at temperature $37 \pm 0.1^{\circ} \mathrm{C}$, was determined according to the following equation:

$f_{\mathrm{AO}}=\frac{\left(S_{\mathrm{AO}}-S_{\mathrm{O}}\right) M_{\text {Trolox }}}{\left(S_{\text {Trolox }}-S_{\mathrm{O}}\right) M_{\mathrm{AO}}} \times f_{\text {Trolox }}$

where $S_{0}, S_{\mathrm{AO}}$ and $S_{\text {Trolox }}$ are the areas, limited by kinetic curves of the fluorescence intensity decrease with and without antioxidants under study as well as the standard antioxidant, trolox, respectively. $M_{\text {Trolox }}$, and $M_{\mathrm{AO}}$ are molar concentrations of trolox and the antioxidant under study; $f_{\text {Trolox }}$ is the antiperoxyradical capacity of trolox, indicating the number of radicals scavenged by one molecule of the antioxidant. Antiperoxyradical capacity in relation to the trolox equivalent (TE), $\left(f_{\text {rel TE }}\right)$, is equal to $\left(f_{\text {rel TE }}=f_{\mathrm{AO}} / f_{\text {Trolox }}\right)$. Integration of the appropriate areas was performed by the method 
of trapezoids using Microcal Origin 8.0 graphic and data analysis software.

In our studies, the necessary conditions for correct calculation of total antiradical capacity by the ORAC method included the presence of distinct induction period of the reaction, and the condition $k_{\mathrm{AO}}[\mathrm{AO}]_{0} \gg k_{\mathrm{FlOH}}[\mathrm{FlOH}]_{0}$ was obeyed. The values of bimolecular rate constants $\left(k_{\mathrm{AO}}\right)$ for the reactions of peroxyl radicals with antioxidants (AO) considered exceeded those of the same radicals at reactions with the fluorescent marker, FL by 1-2 order(s) of magnitude. At the same time, the total initial concentration of the antioxidants was 10 times greater against the initial concentration of fluorescein.

Simultaneously, such values of peroxyl radicals generation rates (reaction (1)) were used that allowed to ignore the reactions between peroxyl radicals (reactions $(2,3)$ ). This is realized at condition $2 \sqrt{k_{\mathrm{ROO}+}+\mathrm{ROO} \cdot \times R_{\mathrm{i}}} \ll f \times k_{\mathrm{AO}}[\mathrm{AO}]_{0}$, where $k_{\mathrm{ROO}+\mathrm{ROO}}$. is the rate constant at the interaction between peroxyl radicals, $k_{\mathrm{AO}}$ is the reaction rate constant of peroxyl radicals with an antioxidant, $R_{\mathrm{i}}$ is the rate of radical initiation at concentration $[\mathrm{AAPH}]_{0}=1.73 \times 10^{-3} \mathrm{M}$ and $T=37 \pm 0.1^{\circ} \mathrm{C}$. For the magnitudes $k_{\mathrm{ROO}+\mathrm{ROO}}=$ $2 \times 10^{4} \mathrm{M}^{-1} \mathrm{c}^{-1}, R_{\mathrm{i}}=2.35 \times 10^{-9} \mathrm{Mc}^{-1}, f=2$, $k_{\mathrm{AO}}=10^{5} \div 2 \times 10^{6}$ the inequality $9.7 \times 10^{-3} \ll 1 \div 20$ is complied for the tertiary peroxyl radicals, ${ }^{23,24}$ that is, the above-mentioned inequality is observed. It follows from this that under the considered conditions the role of alkoxyl radical formation in the reaction between peroxyl radicals is negligibly small. So, the antiradical capacity of binary mixtures of antioxidants is measured in relation to peroxyl radicals.

$$
\mathrm{ROO}+\mathrm{ROO} \rightarrow[\mathrm{ROOOOR}] \coprod_{\mathrm{ROOR}+\mathrm{O}_{2}}^{2 \mathrm{RO} \cdot+\mathrm{O}_{2}}
$$

for $10 \mathrm{~min}$. Kinetic studies by the SWV method were carried out as follows. During the reaction, a decrease in the values of current for characteristic peaks in the voltammograms of individual antioxidants were registered periodically. Kinetic studies were carried out in the phosphate buffer at the same concentrations of the initial reagents as in ORAC experiments at FL absence and saturation of the solution by air.

\section{Results and Discussion}

Figure $2 \mathrm{~A}$ and $2 \mathrm{~B}$ demonstrate experimental results obtained via ORAC method. That is, kinetic curves of fluorescein consumption in the reaction with peroxyl radicals were generated by thermal decomposition of the water-soluble azo initiator AAPH in the dioxygensaturated solution in the presence and absence of antioxidants.

Antiperoxyradical capacities (in the trolox equivalent) of both individual antioxidants and their mixtures were calculated on the basis of kinetic data of FL consumption (Figure 2A and 2B).

The value of non-additive effect (NAE) for a binary mixture of antioxidants was calculated by the formula:

$\mathrm{NAE}=\frac{f_{\mathrm{mix}}-\left(f_{\mathrm{FIOH}}+f_{\mathrm{A}}\right)}{f_{\mathrm{FIOH}}+f_{\mathrm{A}}} \times 100 \%$, 2.2c SWV studies: SWV measurements were performed using "Bioanalytical Systems 100B/W" (BAS, USA). SWV voltammograms were recorded by means of a three-electrode scheme. The working electrode was a glassy carbon electrode with an active geometrically measured surface area of $0.09 \mathrm{~cm}^{2}$. Before each measurement, the last was cleaned with $\mathrm{Al}_{2} \mathrm{O}_{3}$ powder with particle size $0.5 \mu \mathrm{m}$ for $3 \mathrm{~min}$. $\mathrm{Ag} / \mathrm{AgCl}$ served as the reference electrode and a platinum wire was the counter electrode. Phosphate buffer with a concentration of $0.1 \mathrm{M}(\mathrm{pH}=7.4)$ was used as a supporting electrolyte. Operation of the electrochemical analytic system was checked by the standard solution of $\left[\mathrm{Fe}(\mathrm{CN})_{6}\right]^{-3}$, with 0.9995 linear correlation factor at calibration. The volume of the tested solution was $3 \mathrm{~mL}$ at $37 \pm 0.1^{\circ} \mathrm{C}$, the scanning frequency was $25 \mathrm{~Hz}$ and square-wave amplitude was $25 \mathrm{mV}$. SWV voltammograms were registered within the potential interval $0 \div+1600 \mathrm{mV}$. Before each measurement, the solution under study was saturated by molecular nitrogen $(99.99 \%)$ where $f_{\text {mix }}, f_{\mathrm{FlOH}}, f_{\mathrm{A}}$ is antiperoxyradical capacities of the mixture of antioxidants, individual flavonoid, and co-antioxidant, respectively.

As can be seen from the kinetic curves presented in Figure 2A, the rate of FL fluorescence intensity is falling in the presence of the trolox-rutin pair in the reaction mixture. Consequently, the time interval of FL consumption in the presence of antioxidants becomes longer, which is a prediction of the synergistic effect.

On the contrary, in the presence of the quercetintrolox pair in the reaction mixture (kinetic curves of the Figure 2B), the rate of FL fluorescence intensity decrease is growing. Simultaneously, the time intervals of FL consumption in the presence of antioxidants in the reaction mixture is reducing, thus indicating the antagonistic effect. 

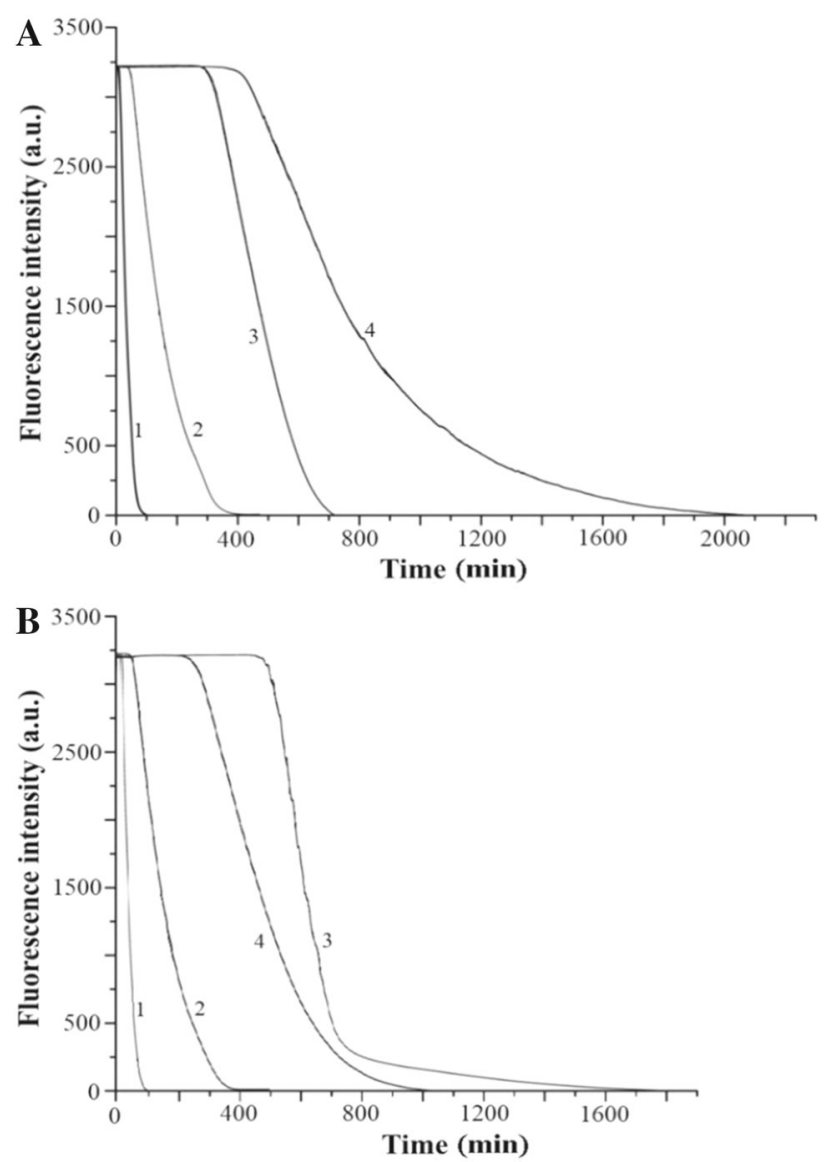

Figure 2. Kinetic curves of FL fluorescence intensity decrease at $515 \mathrm{~nm}$ in reaction with peroxyl radicals in the absence (1) and in the presence (2-4) of antioxidants. (2A): trolox (2), rutin (3), rutin-trolox mixture (4). (2B): trolox (2), quercetin (3), qurcetin-trolox mixture (4). $[\mathrm{AAPH}]_{0}=1.73 \times 10^{-3} \mathrm{M}$, (overall rate of $\mathrm{ROO}^{\bullet}$ production $\left.R_{\mathrm{i}}=2.35 \times 10^{-9} \mathrm{Ms}^{-1}\right)$, concentration of antioxidants: $5 \times 10^{-6} \mathrm{M}$ (separately and in mixture), $[\mathrm{FL}]_{0}=5 \times 10^{-7} \mathrm{M}$. Solvent: deionized water, $\mathrm{T}=37 \pm 0.1^{\circ} \mathrm{C}$.

The values of antiperoxyradical capacities of individual antioxidants and their binary mixtures are summarized in Table 1.

The values of bond dissociation energy (BDE) of the most reactive $\mathrm{OH}$ bond ${ }^{30,31}$ and those of anodic oxidation potential peaks of studied antioxidants, registered by the SWV method for the same antioxidants are presented in Table 2.
1. In the mixtures of O-glucosylated flavonoids (rutin and naringin) with trolox or ascorbic acid positive non-additive co-antioxidant effect, synergism, is observed.

2. In the mixtures of non-O-glucosylated flavonoids (quercetin and morin) with trolox or ascorbic acid negative non-additive antiperoxyradical effect, antagonism, is observed.

3. The value of oxidation potential of flavonoids and $\mathrm{BDE}$ of the most reactive $\mathrm{OH}$ bond is relatively high when glycoside is present in the structure.

4. Synergy is registered in the mixture of trolox and ascorbic acid, which is consonant with the results on the antiradical effect of the mixture of ascorbic acid with $\alpha$-tocopherol: a structural lipophile analog of trolox.

As mentioned above, the value of oxidation potential of flavonoids and $\mathrm{BDE}$ of the most reactive $\mathrm{OH}$ bond is relatively high when glycoside is present in the structure. We suggest that this fact is conditioned by the presence of intramolecular hydrogen bonds between hydrogen atoms and phenolic $\mathrm{OH}$ groups responsible for interaction with radicals and two-electron-donor oxygen atoms of the glucosyl substituent. It is well-known that hydrogen bond formation with the participation of hydrogen atom of the phenolic $\mathrm{OH}$ group leads to diminishing the reactivity relative to the free radicals, simultaneously increasing the value of oxidation potential. ${ }^{12,25,32-35}$ According to the quantum-chemical calculations, ${ }^{36}$ decreasing BDE frequently correlates with the oxidation potential of catecholic $\mathrm{OH}$ groups, which is associated with sterical hindrance induced by glucosyl group for these hydroxyl groups. It follows from Table 2 that at identical structures of flavonoids (quercetin and rutin) the presence of O-glucosyl at rutin leads to a decrease in antiperoxyradical capacity. As a whole, it is well-known that glucosylation of the 3$\mathrm{OH}$ group greatly reduces the antioxidant activity of flavonoids. ${ }^{36-41}$ The SWV signals of quercetin and rutin are most intensive and are associated with reversible two-electron oxidation of catecholic hydroxyl groups of the $\mathrm{B}$ ring according to Scheme $4 .{ }^{41,42}$<smiles>CC1=CC(=O)[C@@H](C=Cc2cc(C)cc(O)c2O)C=C1</smiles>

The following regularities may be selected from the data presented in Tables 1 and 2:
In the initial $5 \times 10^{-6} \mathrm{M}$ concentration of the antioxidants, only for the quercetin-trolox mixture 
Table 1. Antiperoxyradical capacities of antioxidants and their binary mixtures in trolox equivalent as well as the degree of co-antioxidant effect (synergism, antagonism) determined by ORAC method. In brackets, the sum of co-antioxidants $f_{\mathrm{A}}$ is presented.

\begin{tabular}{|c|c|c|c|c|c|}
\hline \multirow[t]{2}{*}{ Antioxidant } & \multirow[t]{2}{*}{$f_{\mathrm{A}}$} & \multirow[t]{2}{*}{$f_{(\mathrm{AO}+\text { Trolox })}$} & \multirow[t]{2}{*}{$f(\mathrm{AO}+\mathrm{AA})$} & \multicolumn{2}{|c|}{$\%$ non-additive effect, with } \\
\hline & & & & Trolox & AA \\
\hline Rutin & 3.80 & $5.64(>4.80)$ & $5.72(>4.78)$ & 17.5 & 19.6 \\
\hline Quercetin & 5.45 & $3.82(<6.45)$ & $5.06(<6.43)$ & -40.7 & -21.3 \\
\hline Morin & 3.19 & $3.56(<4.19)$ & $4.02(<4.17)$ & -15.0 & -3.5 \\
\hline Naringin & 4.11 & $5.48(>5.11)$ & $5.60(>5.09)$ & 7.2 & 10.0 \\
\hline Ascorbic acid & 0.98 & 2.38 & - & 20.2 & - \\
\hline Trolox & 1 & - & 2.38 & - & 20.2 \\
\hline
\end{tabular}

Table 2. Oxidation potentials of antioxidants in relation to $\mathrm{Ag} / \mathrm{AgCl}$ determined by SWV method and BDE values ${ }^{30}$ of the most active radicals for neutralization of radicals of phenolic $\mathrm{OH}$ groups calculated by PM6 quantum-chemical method. $E p_{1}$ and $E p_{2}$ are the first and second oxidation peaks, respectively.

\begin{tabular}{lcccc}
\hline Antioxidant & \multicolumn{2}{c}{ Anodic oxidation potentials, $\mathrm{mV}$} & \multirow{2}{*}{$\mathrm{BDE} \mathrm{kJ} / \mathrm{mol}$} & Most active antiradical site \\
\cline { 2 - 3 } & $E p_{1}$ & $E p_{2}$ & & \\
\hline Rutin & 230 & - & 311.4 & $4^{\prime}-\mathrm{OH}$ \\
Quercetin & 115 & 235 & 295.7 & $4^{\prime}-\mathrm{OH}$ \\
Morin & 160 & - & 322.0 & $3-\mathrm{OH}$ \\
Naringin & 680 & 930 & 337.9 & $4^{\prime}-\mathrm{OH}$ \\
Ascorbic acid & 220 & - & - & - \\
Trolox & 124 & - & 312.1 & OH \\
\hline
\end{tabular}

under studied conditions, it was possible to track simultaneously the consumption of both the antioxidants (Figure 3A). In other cases, the kinetics of flavonoid (quercetin and morin) consumption was determined, conditioned by different voltammetric sensitivity relative to antioxidants, and in some cases by overlapping oxidation peaks at different current intensity.

The results shown in Figure 3A reflect that the time of practically complete consumption of trolox in the absence of rutin $(\sim 38 \mathrm{~min})$ coincides with the induction period of fluorescein consumption in the presence of trolox ( $\sim 40 \mathrm{~min}$, Figure 2$)$, registered by the ORAC method. This clearly points to the accordance of the data obtained by the ORAC method and kinetic SWV studies.

It is seen from Figure 3A (curve 2) that the introduction of trolox into the reaction mixture leads to a decrease in rutin consumption. A similar picture was observed for the rutin - ascorbic acid mixture and at this acceleration of trolox consumption was registered. Most likely, the increase in trolox consumption rate and corresponding retardation in rutin concentration decrease are the causes for the synergic effect of the antioxidants mixture.

In the case of the structural analog of quercetin not containing glucosyl, we succeeded in studying the consumption kinetics by the SWV method only for quercetin. The addition of co-antioxidants, ascorbic acid and trolox (Figure 3B, curves 2 and 3), result in the acceleration of quercetin consumption. This process is likely the reason for the antagonistic effect registered for mixtures of quercetin with trolox and ascorbic acid.

Due to the presence of glucosyl substituent in the flavonoid (rutin and naringin) molecular structure, the synergic effect is observed in their mixtures with coantioxidants trolox and ascorbic acid. Simultaneously, as it follows from Table 2, the values of BDE for the most reactive phenolic $\mathrm{OH}$ groups and oxidation potential are greater in these compounds as compared to other antioxidants. This leads to a diminishing antiradical and corresponding antioxidant abilities of flavonoids. By the example of rutin, the reaction occurring by the mechanism of Hydrogen Atom Transfer (HAT) with peroxyl radicals $\left(\mathrm{ROO}^{\bullet} \equiv \mathrm{LOO}^{\bullet}\right)$ : chain carriers of lipid peroxidation is suggested to be a reaction (5). The HAT mechanism suggests that phenol donates a hydrogen atom to a radical directly, resulting in corresponding phenoxyl radical. At the same time, according to the literary data ${ }^{42,43}$ at the HAT mechanism the intramolecular hydrogen bond (HB) formed between the hydrogen atom of hydroxyl group and the oxygen atom is strengthened by $21.8 \mathrm{~kJ} / \mathrm{mol}$. This magnitude serves as a quantitative measure for ortho-hydroxyl electronic effect resulting in an increase in the reactivity of catecholic $\mathrm{OH}$ groups. 


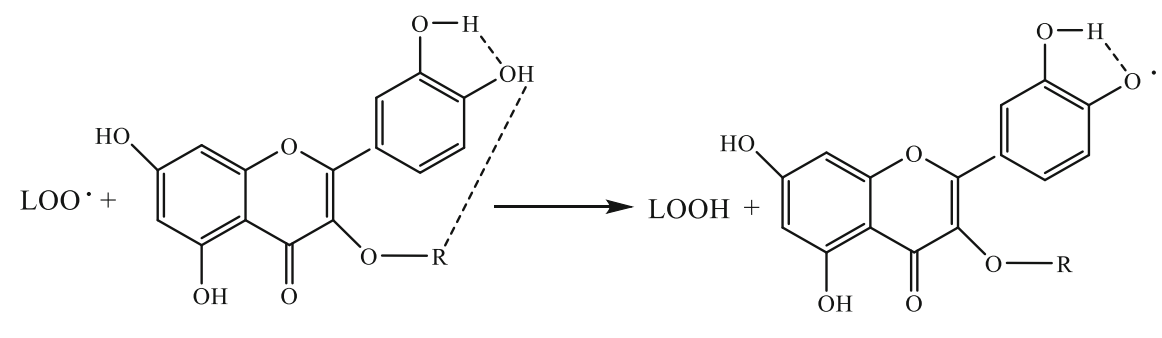

stronger $\mathrm{HB}$ between $\mathrm{H}-\mathrm{O}$ atoms

It should be mentioned that the reaction (5) might proceed via several mechanisms including one-step HAT, as well as multistep mechanisms, where proton and electron transfers occur separately, and by two sequential steps such as Sequential Proton-Loss Electron-Transfer (SPLET) mechanism. ${ }^{28,44,45}$ The SPLET mechanism is described by the following reactions between phenolic substances and free radicals:

$$
\begin{aligned}
& \mathrm{PhOH} \leftrightarrow \mathrm{PhO}^{-}+\mathrm{H}^{+} \\
& \mathrm{PhO}^{-}+\mathrm{ROO} \cdot \rightarrow \mathrm{PhO} \cdot+\mathrm{LOO}^{-} \\
& \mathrm{LOO}^{-}+\mathrm{H}^{+} \rightarrow \mathrm{LOOH}
\end{aligned}
$$

According to this mechanism, phenoxide anion formed in the equilibrium process of the initial phenol deprotonation participates in the reaction with radicals.

Nevertheless, using only HAT mechanism in many cases is preferred due to the following reasons:

1) According to data in Table 2, the correlation between the BDE value ${ }^{30}$ of the weakest phenolic $\mathrm{OH}$ bond and those of the first and second oxidation peaks is observed. As that BDE is based on the singlestep HAT mechanism, the electrochemical oxidation according to reaction (4) is in principle close to a process realized by SPLET mechanism.

2) Quantum-chemical calculations carried out by PM6 method $^{30,31}$ demonstrate the universality of BDE values for flavonoids that determine their antiradical activity despite the mechanism of free radical hydrogen atom abstraction from phenolic $\mathrm{OH}$ group of the antioxidant. The authors reason this phenomenon by the same change in the value of reaction enthalpy for HAT and SPLET mechanisms.

Primary antiradical activity of a flavonoid is controlled by the HAT mechanism thermodynamically based on BDE values of the hydroxyl groups. That is, at first, hydroxyl groups with small BDE values react with peroxyl radicals. According to the data ${ }^{46}$ obtained by DFT (Density Functional Theory), BDE of quercetin's $\mathrm{OH}$ groups change in the following sequence:

$4^{\prime}-\mathrm{OH}<3^{\prime}-\mathrm{OH}<3-\mathrm{OH}<7-\mathrm{OH}<5-\mathrm{OH}$
It is particularly remarkable that the rate of rutin consumption is considerably lower as compared to its molecular analog, quercetin, not containing glucosyl substituent in the molecular structure (Figure 3A and $3 \mathrm{~B})$. This is not associated with incomplete scavenging of the generated peroxyl radicals by rutin because of high BDE of phenolic $\mathrm{OH}$ groups. The rate of rutin consumption is practically constant at increasing its initial concentration (Figure 3C). This indicates to the fact that all generating peroxyl radicals are completely scavenged by rutin.

According to literature data, ${ }^{38} 3$-O-glucoside group substituent contained in the rutin molecule may participate in the HAT intramolecular reaction leading to a reduction of the catecholic hydroxyl groups. This leads to deceleration of the rutin oxidation rate registered analytically in our experiments SWV (Figure 3A).

The participation of fluorescein, a phenolic compound, in the total non-additive effect of the two observable antioxidants is not taken into consideration because of far less antioxidant activity of Fl, as well as due to its low concentrations (10 times) in the initial reaction mixture as compared to those of the studied antioxidants. The validity of such an approach is confirmed by a good fit of ORAC data with those of kinetic electrochemical studies by the SWV method, where fluorescein with phenolic structure was absent.

Considering the 'skeletal' kinetic models of the reaction leads to the explanation of the phenomena of synergy and antagonism for the mixture of trolox (TH) with flavonoids (FlOH). In this case, it is suggested that the $\mathrm{OH}$ group of the flavonoid with the smallest BDE participates in the reaction.

The following kinetic model explains the antioxidant synergic effect of binary mixtures of rutin or naringin with trolox.

$$
\begin{aligned}
& \mathrm{LOO}^{\bullet}+\mathrm{TH} \rightarrow \mathrm{LOOH}+\mathrm{T}^{\bullet} \\
& \mathrm{T}^{\bullet}+\mathrm{LOO}^{\bullet} \rightarrow \mathrm{LOOT} \\
& \mathrm{LOO}^{\bullet}+\mathrm{FlOH} \rightarrow \mathrm{LOOH}+\mathrm{FlO}^{\bullet} \\
& \mathrm{FlO}^{\bullet}+\mathrm{TH} \rightarrow \mathrm{FlOH}+\mathrm{T}^{\bullet} \quad \text { (drive forces) }
\end{aligned}
$$$$
\mathrm{FlO}^{\bullet}+\mathrm{LOO}^{\bullet} \rightarrow \text { non radical products (drive forces) }
$$ 

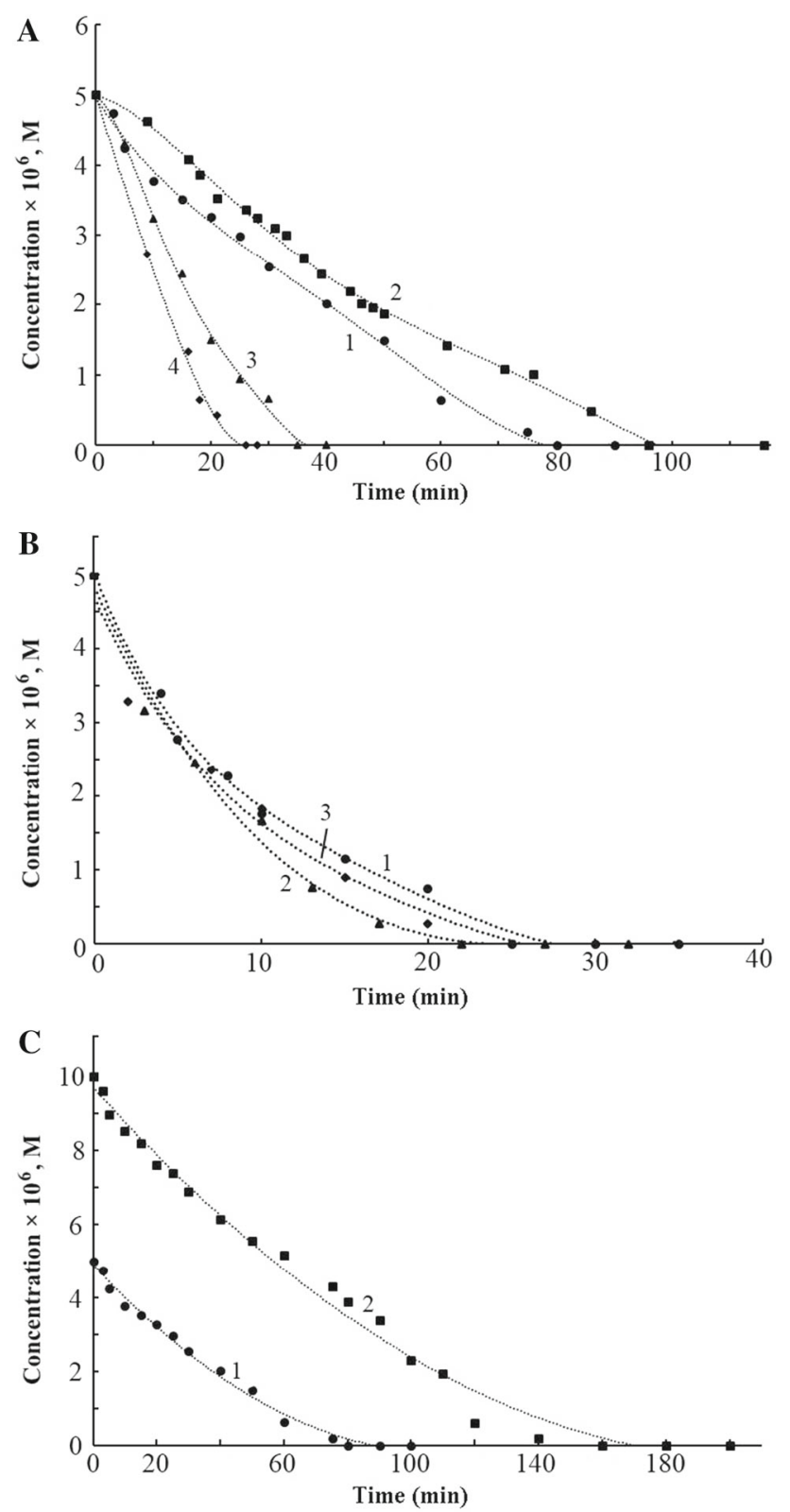

Figure 3. Kinetic curves of antioxidant consumption. $[\mathrm{AAPH}]_{0}=1.73 \times 10^{-3} \mathrm{M},[\mathrm{Fl}]_{0}=5 \times 10^{-7} \mathrm{M}$, phosphate buffer $0.1 \mathrm{M}, \mathrm{pH}=7.4$. (3A). Rutin in the absence (1) and presence (2) of trolox, and trolox in the absence (3) and presence (4) of rutin. (3B). Quercetin in the absence (1) and the presence of trolox (2) and ascorbic acid (3). (3C). Rutin $(1,2)$ at its different initial concentrations and absence of co-antioxidant, $\mathrm{T}=37 \pm 0.1^{\circ} \mathrm{C}$.

This kinetic model, relevant to the synergy of the antioxidants combined effect, explains the reason for more rapid consumption of trolox in the mixture. In the reaction, trolox consumption starts (Figure 2A) due to the stronger bond in the flavonoid's $\mathrm{OH}$ group. Simultaneously, peroxyl radical reacts with flavonoid by the reaction (11) yielding a phenoxyl radical, FlO'. The reaction (12) between phenoxyl radical $\mathrm{FlO}^{\bullet}$ and trolox, resulting in reproduction of the initial flavonoid, explains acceleration in trolox consumption and deceleration in flavonoid consumption. This is in compliance with the results, where electrochemical study combined with ESR spectroscopy showed that flavonoids were regenerated from aroxyl radicals by $\alpha$-tocopherol under certain conditions. ${ }^{47}$

The synergy of the co-antioxidants mixture is associated with the following fact. After 20 min from the initial reaction when trolox is practically absent in the reaction mixture, the consumption process of the analytically registered by the SWV method catecholic $\mathrm{OH}$ groups of the $\mathrm{B}$ ring in flavonoids takes approximately $120 \mathrm{~min}$ (Figure 3A) according to kinetic data. In ORAC experiments, consumption time of the initial flavonoid is considerably longer because of further participation of catecholic $\mathrm{OH}$ groups of the $\mathrm{B}$ ring, as well as hydroxyl groups of $\mathrm{A}$ and $\mathrm{C}$ rings in the reaction. Experimental data shown in Figure 4 testifies in this case about the consecutive transformation of phenolic $\mathrm{OH}$ groups of the flavonoids. Early in the occurring reaction, the intensity of the oxidation peak $E p_{1}=115 \pm 15 \mathrm{mV}$ decreases. At the second oxidation peak $E p_{2}=235 \pm 20 \mathrm{mV}$, there appears an overlap by the first peak. Then the second oxidation peak decreases as a result of interaction with peroxyl radicals. In the process when trolox is already consumed, the concentration of the flavonoid, registered at that moment, turns to be higher as compared to the same instant of time when trolox is absent in the initial mixture (Figure 3A). This is the reason for the prolonged inhibition of fluorescein consumption in ORAC experiments resulting in the observed synergic effect.

The kinetic model given below explains the antioxidant antagonistic effect of binary mixtures of quercetin or morin with trolox. The first three main reactions, (9), (10) and (11) are the same as in the model for the synergic effect. The following two reactions responsible for the antagonistic effect result in the main difference between the presented two kinetic models:

$\mathrm{T}^{\bullet}+\mathrm{FlOH} \rightarrow \mathrm{FlO}^{\bullet}+\mathrm{TH} \quad$ (drive forces)

$\mathrm{FlO}^{\bullet}+\mathrm{T}^{\bullet} \rightarrow$ non radical products $\quad$ (drive forces)

According to the kinetic model, in explaining antagonistic effect of co-antioxidants, tocopheroxyl radical $(\mathrm{T} *)$ actively participates in the HAT reaction (14) with quercetin and morin, accelerating their consumption (Figure 3B). This is conditioned by the presence of more reactive catecholic dihydoxyl groups in the $\mathrm{B}$ ring with smaller BDE (Table 1). 


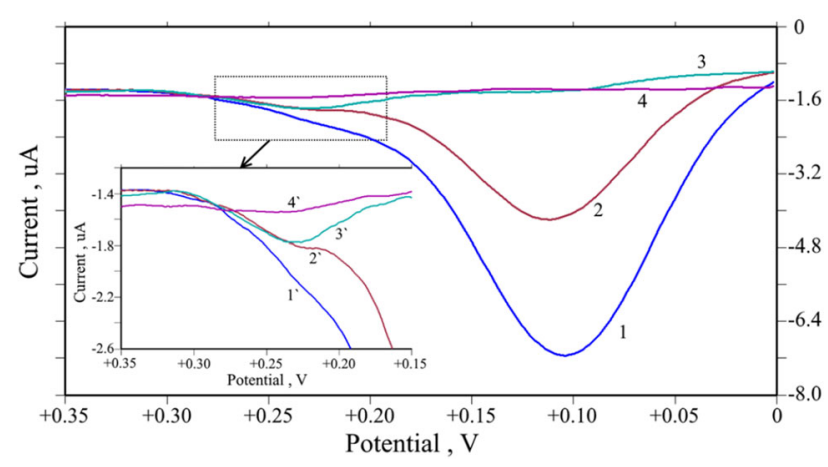

Figure 4. The changes in the intensity of oxidation peaks $\left(E p_{1}=115 \pm 15 \mathrm{mV}\right.$ and $\left.E p_{2}=235 \pm 20 \mathrm{mV}\right)$ for quercetin at reaction times: $0 \mathrm{~min}\left(1\right.$ and $\left.1^{\prime}\right), 12 \mathrm{~min}\left(2\right.$ and $\left.2^{\prime}\right), 20 \mathrm{~min}$ $\left(3\right.$ and $\left.3^{\prime}\right)$, $120 \mathrm{~min}\left(4\right.$ and $\left.4^{\prime}\right)$. The second oxidation peak $\left(E p_{2}=235 \pm 20 \mathrm{mV}\right)$ appears after the decrease of the first more intensive peak. [Quercetin] $0=5 \times 10^{-6} \mathrm{M}$, $[\mathrm{AAPH}]_{0}=1.73 \times 10^{-3} \mathrm{M}$, in phosphate buffer $\mathrm{pH}=7.4$.

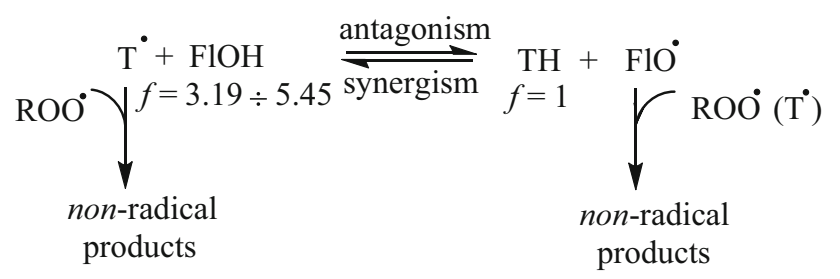

Figure 5. The reaction scheme demonstrating non-additive effects in binary mixtures of co-antioxidants flavonoids with trolox.

Antagonism observed in ORAC experiments can most likely be associated with the following reasons: (i) after trolox consumption, a smaller flavonoid concentration is registered vis-à-vis the same timepoint in the absence of trolox in the initial mixture (Figure 3B); (ii) in the initial stage when trolox is present in the reaction mixture, the formed tocopheroxyl radical actively participates in reaction (14) and in the cross-reaction (15) with $\mathrm{FlO}^{\bullet}$ radical. These reactions compete with the reaction (10) by which radical $\mathrm{T}^{\bullet}$ traps the generating peroxyl radical. As a result, antiperoxyradical capacities of the binary mixtures of co-antioxidants decrease. Simultaneously, antioxidant FlOH with high antiradical capacity is consumed by reactions (14) and (15). Meanwhile, regeneration of trolox by reaction (14) with considerably smaller antiradical capacity in relation to flavonoids does not compensate the acceleration (increasing rate) of the flavonoids consumption. Such reactions and synergic and antagonistic effects by the same scheme also take place in the case of ascorbic acid (when replacing trolox) in binary mixtures with flavonoids.
In general, non-additive effects in binary mixtures of antioxidants are primarily conditioned by the possibility of shift the quasi-equilibrium with the participation of phenoxyl radicals (Figure 5) in the direction of regeneration of an antioxidant with high (synergy) or low (antagonism) antiperoxyradical capacity, respectively.

\section{Conclusions}

Using ORAC and SWV methods non-additive effect was characterized by the values of antiperoxyradical capacities of antioxidants and their mixtures determined in trolox equivalency.

In the mixtures with trolox or ascorbic acid, a much more pronounced synergistic effect is observed for flavonoids (rutin and naringin) having glucosyl groups in the molecular structure - relatively high oxidation potential and $\mathrm{BDE}$ of the most reactive $\mathrm{OH}$ group. In the offered kinetic model in the mixtures containing trolox, the peroxy radical attacks $\mathrm{OH}$ group of the flavonoid yielding a relatively reactive phenoxyl radical. This radical reacts with trolox resulting in regeneration of the initial flavonoid with high antiradical capacity.

The antagonistic effect is observed when flavonoids with small oxidation potential and the BDE of the most reactive $\mathrm{OH}$ groups, in particular, flavonoids without glucosyl group, are present in the mixture. If trolox is present in the mixture, the formed tocopheroxyl radical $\mathrm{T}^{\bullet}$ reacts with the more reactive phenolic $\mathrm{OH}$ group of the flavonoid. Therefore, after a relatively fast consumption of trolox, the concentration of the flavonoid becomes lower than at the same time point at the absence of trolox in the initial mixture. Thus, the antioxidant effect is lower after trolox consumption.

Integrally, synergistic and antagonistic effects of flavonoids with trolox or ascorbic acid in a binary mixture are conditioned by competing cross-reactions of hydrogen atom abstraction from the co-antioxidant molecules by phenoxyl radicals. In the case of the predominating reaction of antioxidant regeneration with high antiradical capacity, the synergistic phenomenon is observed. The antagonism is observed in the case of the predominating reaction of antioxidant regeneration with lower antiradical capacity.

\section{Acknowledgements}

This work is financed by the Committee of Science of Armenia (Basic Research program). The authors thank Mr. G. Kocharyan (IChPh NAS Armenia) for technical assistance. 


\section{Compliance with ethical standards}

Conflict of interest None.

Compliance with ethics requirements This article does not contain any studies with human or animal subjects.

\section{References}

1. Embuscado M E 2015 Spices and herbs: natural sources of antioxidants - a mini review J. Funct. Food. Part B 18 811

2. Oroian M and Escriche I 2015 Antioxidants: characterization, natural sources, extraction and analysis Food Res. Int. 7410

3. Shahidi F and Ambigaipalan P 2015 Phenolics and polyphenolics in foods, beverages and spices: antioxidant activity and health effects_a review J. Funct. Food. Part B 18820

4. Rice-Evans C A, Miller N J and Paganga G 1997 Antioxidant properties of phenolic compounds Trends Plant Sci. 2152

5. Hotta H, Nagano S, Ueda M, Tsujino Y, Koyama J and Osakai T 2002 Higher radical scavenging activities of polyphenolic antioxidants can be ascribed to chemical reactions following their oxidation Biochemic. Biophys. Acta 1572123

6. Masuoka N, Matsuda M and Kubo I 2012 Characterisation of the antioxidant activity of flavonoids Food Chem. 131541

7. Jovanovic S V, Steenken S, Tosic M, Marjanovic M and Simic M G 1994 Flavonoids as antioxidants J. Am. Chem. Soc. 1164846

8. Pietta P-G 2000 Flavonoids as antioxidants J. Nat. Prod. 631035

9. van Acker S A B E, van den Berg D J, Tromp M N J L, Griffioen D H, van Bennekom W P, van der Vijgh W J F and Bast A 1996 Structural aspects of antioxidant activity of flavonoids Free Radic. Biol. Med. 20331

10. Xiao Z-P, Peng Z-Y, Peng M-J, Yan W-B, Ouyang Y-Z and Zhu H-L 2011 Flavonoids health benefits and their molecular mechanism Mini Rev. Med. Chem. 11169

11. Chen A Y and Chen Y C 2013 A review of the dietary flavonoid, kaempferol on human health and cancer chemoprevention Food Chem. 1382099

12. Ingold K U and Pratt D A 2014 Advances in radicaltrapping antioxidant chemistry in the 21st century: a kinetics and mechanisms perspective Chem. Rev. 114 9022

13. Wang S, Meckling K A, Marcone M F, Kakuda Y and Tsao R 2011 Sinergistic, additive and antagonistic effects of food mixtures on total antioxidant capacities J. Agric. Food Chem. 59960

14. Niki E, Saito T, Kawakami A and Kamiya Y 1984 Inhibition of oxidation of methyl linoleate in solution by vitamin E and vitamin C J. Biol. Chem. 2594177

15. Karpukhina G V and Emanuel N M 1984 Classification of synergistic mixtures of antioxidants and the mechanisms of synergy Dokladi Akademii Nauk SSSR 2761163 (In Russian)
16. Denisov E T and Azatyan V V 2000 Inhibition of Chain Reactions (London: Gordon and Breach) p. 338

17. Pedrielli P and Skibsted L H 2002 Antioxidant synergy and regeneration effect of quercetin, (-) epicatechin, and $(+)$ catechin on $\alpha$-tocopherol in homogenous solutions of peroxidating methyl linoleate J. Agric. Food Chem. 507138

18. Colon M and Nerin C 2016 Synergistic, antagonistic and additive interactions of green tea polyphenols Eur. Food Res. Technol. 242211

19. Wei Q-Y, Zhou B, Cai Y-J, Yang L and Liu Z-L 2006 Synergistic effect of green tea polyphenols with trolox on free radical-induced oxidative DNA damage Food Chem. 9690

20. Nogala-Kałucka M, Dwiecki K, Siger A, Górnaś P, Polewski K and Ciosek S 2013 Antioxidant synergism and antagonism between Tocotrienols, quercetin and rutin in model system Acta Aliment. 42360

21. Wang S and Zhu F 2015 Dietary antioxidant synergy in chemical and biological systems Crit. Rev. Food Sci. Nutr. 572343

22. Niki E 1987 Interaction of ascorbate and $\alpha$-tocopherol Ann. N. Y. Acad. Sci. 498186

23. Kocharyan $\mathrm{G} \mathrm{H}$, Minasyan $\mathrm{S} \mathrm{H}$, Manukyan $\mathrm{Z} \mathrm{H}$ and Tavadyan L A 2016 The synergestic and antagonistic effects of antiperoxyradical activities of flavonoid and trolox mixtures in water medium Chem. J. Armen. 6922 (in Russian)

24. Kocharyan G H 2015 The synergetic and antagonistic effects of antiperoxyradical activities of flavonoid and ascorbic acid mixtures Proced. Eng. Acad. Armen. 12 762 (in Armenian)

25. Foti M C 2007 Antioxidant properties of phenols $J$. Pharm. Pharmacol. 591673

26. Parker T L, Miller S A, Myers L E, Miguez F E and Engeseth N J 2010 Evaluation of synergistic antioxidant potential of complex mixtures using oxygen radical absorbance capacity (ORAC) and electron paramagnetic resonance (EPR) J. Agric. Food Chem. 58 209

27. Gao G, Alessio H M and Cutler R G 1993 Oxygen-radical absorbance capacity assay for antioxidants Free Radic. Biol. Med. 14303

28. Amorati R and Valgimigli L 2015 Advantages and limitations of common testing methods for antioxidants Free Radic. Res. 49633

29. Gordon A and Ford R 1976 The Satellite of Chemist (MIR, Moscow) pp. 514-541. (in Russian)

30. Amic D, Stepanic V, Lucic B, Markovic Z and Dimitric Markovic J M 2013 PM6 study of free radical scavenging mechanisms of flavonoids. Why does $\mathrm{O}-\mathrm{H}$ bond dissociation enthalpy effectively represent free radical scavenging activity J. Mol. Model. 192593

31. Stepanić V, Gall Trošelj K, Lučić B, Marković Z and Amić D 2013 Bond dissociation free energy as a general parameter for flavonoid radical scavenging activity Food Chem. 1411562

32. Marteau C, Favier D, Nardello-Rataj V and Aubry J-M 2014 Dramatic solvent effect on the synergy between $\alpha$-tocopherol and BHT antioxidants Food Chem. 160 190 
33. Snelgrove D W, Lusztyk J, Banks J T, Mulder P and Ingold K U 2001 Kinetic solvent effects on hydrogenatom abstractions: Reliable, quantitative predictions via a single empirical equation $1 \mathrm{~J}$. Am. Chem. Soc. 123469

34. Tavadyan L A, Mardoyan V A and Nalbandyan A B 1986 The reactivity of tertiary alkyl peroxyl radicals in liquid phase. The solvent effect on tertiary butylperoxyl radical reaction with benzaldehyde and phenol Chem. Phys. 5 1377 (in Russian)

35. Tavadyan L A, Musaelyan M, Minasyan S H and Greenaway F T 2014 Mediated kinetic medium effect in the reaction of bis-3,5-di-iso-propylsalicylatozinc(II) with tert-butylperoxyl radicals Inorg. Chim. Acta 418119

36. Cai W, Chen Y, Xie L, Zhang H and Hou C 2014 Characterization and density functional theory study of the antioxidant activity of quercetin and its sugar-containing analogues Eur. Food Res. Technol. 238121

37. Panat N A, Amrute B K, Bhattu S, Haram S K, Sharma G K and Ghaskadbi S S 2015 Antioxidant profiling of C3 quercetin glycosides: Quercitrin, Quercetin 3$\beta$-D-glucoside and Quercetin 3-O-(6"-O-malonyl)- $\beta$-Dglucoside in cell free environment Free Radic. Antioxid. 590

38. Kasaikina O T, Kartasheva Z S, Kancheva V D, Yanishlieva N V and Totseva I R 2010 Consumption of quercetin and rutin in reactions with free radicals Bulg. Chem. Commun. 42153

39. Jin $\mathrm{Y}, \mathrm{Lu}$ Y, Han G-Z, Li W-P, Zhou Q, Jin F-X and Yu H-S 2007 Structure-effect study of quercetin and its sugar-containing analogues as antioxidants in in vitro $\mathrm{H}_{2} \mathrm{O}_{2}$-induced cell injury model Asian J. Pharmacokinet. Pharmacodyn. 7295
40. Farkas O, Jakus J and Heberger K 2004 Quantitative structure-antioxidant activity relationship of flavonoid compounds Molecules 91079

41. Brett A M O and Ghica M-E 2003 Electrochemical oxidation of quercetin Electroanalysis $\mathbf{1 5}$ 1745

42. Janeiro P and Brett A M O 2005 Solid state electrochemical oxidation mechanisms of morin in aqueous media Electroanalysis $\mathbf{1 7} 733$

43. Correia C F, Guedes R C, dos Santos R M B, Cabral B J C and Simoes J A M 2004 $\mathrm{OH}$ bond dissociation enthalpies in hydroxyphenols. A time-resolved photoacousticcalorimetry and quantum chemistry study Phys. Chem. Chem. Phys. 6 2109

44. Litwinienko G and Ingold K U 2007 Solvent effects on the rates and mechanisms of reaction of phenols with free radicals Acc. Chem. Res. 40 222

45. Di Meo F, Lemaur V, Cornil J, Lazzaroni R, Duroux J-L, Olivier Y and Trouillas P 2013 Free radical scavenging by natural polyphenols: atom versus electron transfer $J$. Phys. Chem. A 1172082

46. Rajendran $\mathrm{M}$, Ravichandran $\mathrm{R}$ and Devapiriam D 2013 Electronic description of few selected flavonoids by theoretical study Int. J. Comput. Appl. 77 18

47. Jorgensen L V, Madsen H L, Thomsen M K, Dragsted L O and Skibsted L H 1999 Regeneration of phenolic antioxidants from phenoxyl radicals: an ESR and electrochemical study of antioxidant hierarchy Free Radic. Res. 30207 